\title{
Enhancing Performance Music Games in Education through the IEEE 1599 Standard
}

\author{
Luca Andrea Ludovico, ${ }^{1, *}$, Mario Malcangi ${ }^{1}$, Giuseppina Rita Mangione ${ }^{2}$ \\ ${ }^{1}$ Dipartimento di Informatica, Università degli Studi di Milano, Milan, Italy \\ ${ }^{2}$ Dip. di Ingegneria dell'Informazione, Ingegneria Elettrica e Matematica Applicata, Università degli Studi di Salerno, Salerno, Italy \\ *Corresponding author: ludovico@di.unimi.it
}

Received June 18, 2014; Revised June 27, 2014; Accepted July 02, 2014

\begin{abstract}
This work aims at proposing an innovative way to approach music education, focusing performance games and advanced natural interfaces. The key idea is coupling the power of IEEE 1599, an XML-based international standard for music description, with the concept of music meta-instrument. Meta-instruments are ad hoc interfaces which allow a simplified interaction among the student and music contents. The proposed framework will provide a tool for music practice, powered by the typical features of an IEEE 1599 document. Both a brief overview of the standard and a meta-instrument case study will be presented.
\end{abstract}

Keywords: performance games, music education, IEEE 1599, meta-instruments

Cite This Article: Luca Andrea Ludovico, Mario Malcangi, and Giuseppina Rita Mangione, "Enhancing Performance Music Games in Education through the IEEE 1599 Standard.” American Journal of Educational Research, vol. 2, no. 7 (2014): 486-492. doi: 10.12691/education-2-7-10.

\section{Introduction}

The relevance of new technologies in the educational field - and music learning in particular - is well known and publicly recognized. In spite of a number of solicitations coming from the scientific community of interest, music education field is still lacking of clear guidelines as well as innovative didactic tools able to support evidences - or to confute theories and scenarios about this specific domain [1,2].

The most recent studies recognize the necessity of a better comprehension about the design of learning experiences in music education for the young people, symbionts of new technologies. Music learning needs a deep review of curricula in order to get the integration of multimodal experiences based on simultaneous moving, creating, playing, and reflecting activities [3]. The final goal is obtaining a "symbolically fluent learner" [4,5].

Nowadays music education is renewing its own didactic architectures, rooted in the pedagogic activism principles, in the theories of Schola nova, and in the recent rediscovery of Montessorian and Munarian ideas, coming to the recent Umwelt concepts about body-environment interaction as an educational space $[6,7,8]$. A particularly relevant theory is the one of Embodied and Situated Cognition, which states that Sensory-Motor Interaction (SMI) with the external environment determines the organization of student's neuro-cognitive structures. The purpose is discovering psycho-pedagogic practices to valorize the potential of new technologies and to use them to engage students in the instrumental learning activities [9].
Cognitive situated approach has been recently discussed in the pedagogical field. This theory has its milestone in the scientific work by Montessori, who proposed direct contact and work with objects through their manipulation. Montessori's pedagogical praxis gets benefit from gesture technologies and tactile interfaces, and - recovering Munarian theories - it promotes the idea of an active learning where students are called to intervene and express through music games.

Physical objects expressively designed to support edutainment, such as Froebel-inspired Manipulatives (FiMs) or Montessori-inspired Manipulatives (MiMs), can be rethought in order to model real-world as well as more abstract structures (like music ones). The reason of their success is being oriented to a natural and immediate interaction, by recovering the body element in education either to improve performances or to develop specific skills.

Focusing on information technology, music games are a subset of computer games finalized to enable users to interact with music intuitively, stimulating different types of intelligence, such as the instinctive, intuitive, and sensory-motor one [10]. The application of computer games to music education has been discussed in a number of scientific works - see e.g. [11] - and exploited in many experiments - see e.g. [12].

One of the latest tendencies in computer-assisted music education is the adoption of performance games based on the concept of meta-instrument. As explained in detail below, a meta-instrument is conceived to move some features of music performance into the instrument itself, and to leave others under the performer's control. Scientific literature has discussed the importance of improving control and meta-control processes in this kind of games in order to allow music expressiveness without 
requiring specific expertise. The goal of this work is providing not only an engaging framework, but also an educational tool able to scaffold students' technical skills. Games such as Metapiano or other meta-instruments, described later in the case study section, can be used to extend player's expressive possibilities and to improve self-control and self-regulation at different technical levels. In order to transform the concept of music metainstrument into a device to play intuitively, we propose a framework that embeds meta-instrument notation in the IEEE 1599 format.

Before describing the key features of such a standard and presenting a number of clarifying examples, we will provide a short state of the art about existing performance games. The purpose is making a cluster analysis on the base of technique and expressiveness axes, useful to design and implement a new educational tool valorizing the technical dimension in the context of music curricula renovation.

\section{Music Action Games: State of the Art}

On the marketplace, many music action games have been released. Some of them have become very popular and obtained important commercial results. According to experts, the first music game to be released on a home console was Dance Aerobics (known as Dance Studio in Japan), which dates back to 1987. This precursor of many dance-oriented computer games required players to use the NES Power Pad to mimic an on-screen instructor who moved to the music. Users would have to follow the onscreen characters' moves by stepping on the correct parts of the mat. Dance Aerobics was an early example of rhythm action game.

Rhythm game is a genre of music-themed action video game based on the player's sense of rhythm. Games in the genre typically focus not only on dance, but also on the simulated performance of musical instruments, and require players to press sensitive areas of a device in a sequence shown on the screen. While conventional controllers may be used as input devices, rhythm games often feature pressure-sensitive pads to follow dance movements, ad hoc game controllers that emulate musical instruments, or microphones to catch sound sources. In this sense, it is worth citing blockbusters such as Guitar Hero and SingStar. The recent release of intuitive interfaces (e.g. Nintendo WII Remote and Microsoft Kinect) is leading the way in developing music games more and more engaging. The purpose is improving extragame capacities, and specifically player's musical skills.

The key question is: can this kind of games foster music learning? A Guitar Center survey cited in [13] indicates that $67 \%$ of rhythm players after the game experience will certainly buy a real music instrument. From these results, we can affirm that rhythm games can arouse interest towards the matter. However, if we concentrate on the game itself - here intended as a serious game and not as mere entertainment - the goal should be making the players active actors in the music learning process, providing them with expressiveness and autonomy. Are music games available where expressiveness (a requirement to engage a musician) and instrumental technique aspects (a requirement for music practice and didactics) are balanced?
Reference [13] identifies two key aspects that influence music performance in games: i) the physical-technical dimension and ii) the expressive dimension. As regards the former dimension, the physical aspect is related to the player's morphology and to the type of control over the game. The technical element, instead, can be referred to the interaction of the player with the controller in order to reach specific skill levels.

Expressiveness is an aspect neither physical, nor technical. It can be considered as "the intentional deviations from a reference, i.e. deviation in rhythm, articulation, dynamics or the adding of effects such as vibrato or timbre changes". Expressiveness is allowed in those games providing a certain degree of "musical freedom": e.g. playing notes when no match is proposed, changing the music tempo in real-time, etc. Music elements which can be controlled range from sound production to tempo variations and phrasing.

Nowadays there is a great commercial interest towards musical video games, even if they usually do not guarantee freedom for expressive and technical performance. On one hand, their success among nonprofessional musicians is clear. In 2008, it was reported that music games had become the second most popular video game genre (behind action) in the United States, with $53 \%$ of players being female, above all thanks to the success of the Rock Band and Guitar Hero franchises. On the other hand the opinion on music action games dramatically changes when 10-20 years old music students have been interviewed. In Rock Band or Guitar Hero, for example, the game play requires using drum-like controllers following a fixed schema: adding or modifying the melody or rhythm is considered an error. In this sense, environments such as Wii Music leave more space for action and improvisation.

Needless to say, video gamers and music learners present different expectations: the former category prefers an involving and easy way to approach a music instrument, whereas the latter is searching for a finest control on music parameters. This scientific research has resulted in the design of music environments to foster technical element abstraction by focusing on expressiveness and decreasing the human action frequency.

In the last years, so-called performance games based on the concept of meta-instrument have appeared. A music meta-instrument $[14,15]$ can be either a virtualization of an existing instrument or a brand new one. In both cases, it is conceived to move the sound generation and texture capabilities into the instrument itself, and to leave sequencing and timing under the performer's control.

Relevant examples of performance games are BAO $\mathrm{PAO}$ and Metapiano. BAO PAO is a gestural device developed by Jean Schmutz in cooperation with Jean Haury aiming at encouraging music expressiveness in students with special needs. The user is not allowed to directly produce any sound, since everything is generated by a computer connected with BAO PAO. A music score is pre-loaded and the player can influence the performance by moving a stick across a laser beam. This kind of interaction is an intuitive way to provide expressive interpretation [16].

The Metapiano, designed and developed by Jean Haury, presents a simplified keyboard interface made of only 9 keys. A properly encoded version of a music score is 
provided to the system, and the player can perform score following by pressing any key of the interface. With respect to BAO PAO, a deeper interaction can be achieved, by influencing rhythm, velocity, articulation and so on.

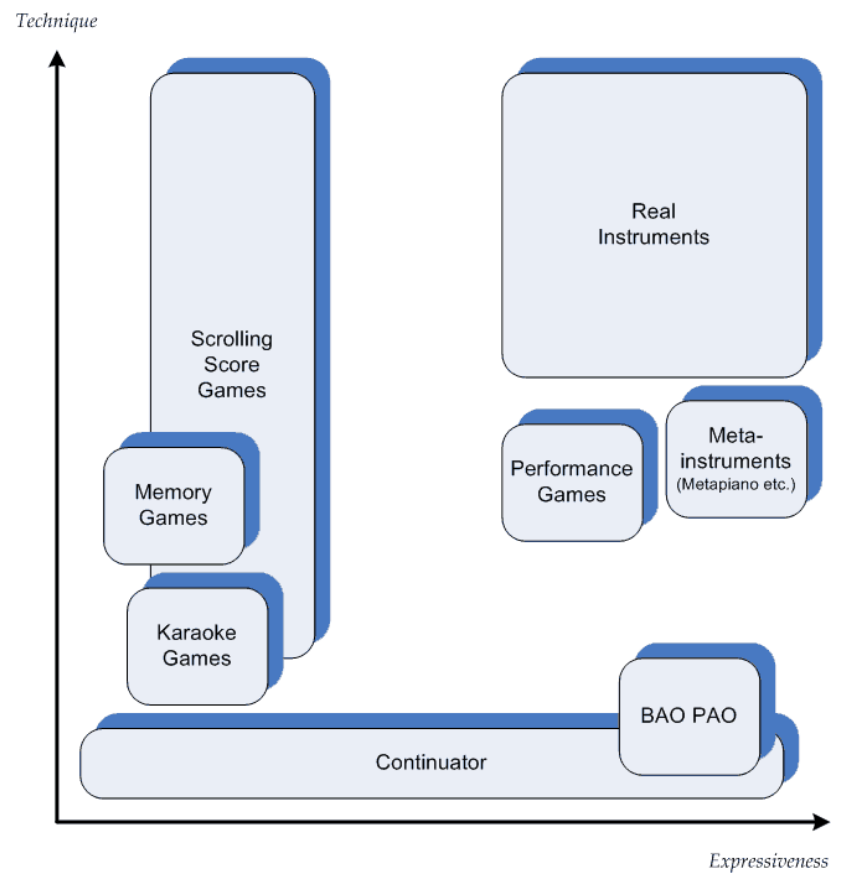

Figure 1. Performance games vs. other music games

Figure 1, an updated version of the one in [13], presents a graphical comparison of the principal music games. This analysis reveals that some performance games privilege expressiveness, others technique, and finally metainstruments try to mix the two approaches, being closer to the real music instruments.

Nowadays, research activities in this field aim at improving both control and meta-control processes, in order to allow an expressive performance without asking the game player to be a virtuoso, but addressing instrumental technical skills too. On one hand, performance-oriented meta-instruments such as the Metapiano can provide a simplification of some performance aspects, and on the other hand they can even extend expressive potentialities through ad-hoc supports and standards. In our opinion a new learning paradigm for music based on advanced performance games is extremely innovative since it could remove any starting barrier between the player and the instrument.

In order to further develop the concept of music metainstrument, in the following we will discuss a framework that embeds meta-instrument notation into the IEEE 1599 format. IEEE 1599 is an XML-based international standard aiming at the representation of music in all its aspects. Before providing details about our proposal and showing a case study, the key concepts of IEEE 1599 will be recalled.

\section{Key Concepts of the IEEE 1599 Standard}

IEEE 1599 is a standard internationally recognized by the IEEE, sponsored by the Computer Society Standards Activity Board and designed by the Technical Committee on Computer Generated Music. IEEE 1599 adopts XML (eXtensible Markup Language) in order to describe a music piece in all its aspects [17]. This challenging goal is achieved through the following concepts:

- A multi-layer layout for content description;

- A common data structure to index and refer to different descriptions of the same music entity, known as the spine;

- Full synchronization among different descriptions of the same music entity.

All the mentioned issues will be briefly discussed in the following subsections. For further details, please refer to [18].

\subsection{Multi-layer Structure}

Music description is a complex task which can embrace a number of different facets. For example, when people address a music work, they usually identify it through commonly-accepted metadata, such as its title, authors, performers, instrumental ensemble, etc.

As regards the visual aspects of music, a piece can be described through notation, i.e. any system that represents scores through the use of written symbols. Depending on cultures, traditions and geographical areas, many methods are in use for notated music, ranging from modern staff notation [19] to neumes [20], from asian solmization [21] to Indian sargam [22], from lute tablature [23] to African dance drumming [24]. Moreover, the same music piece namely the same set of music symbols - can be represented with different page layouts (e.g. different printed editions), or through different kinds of notation (colored music notation, Braille, etc.).

Audio contents represent another kind of description for music. Once again, the same music piece can be performed in many ways, as regards interpretation, score version, ensemble, etc. Depending on the music genre, this fact presents a number of implications: in classical music, even if score symbols are (usually) common to any performance, players adopt their own interpretative model; in jazz, improvisation is a key aspect, so that each performance of a jazz standard becomes unique; in pop/rock music, acoustic versions, covers, etc. are quite common; and so on.

The previous examples intuitively show the richness implied by the locution "music description". The number of heterogeneous ways to describe music is potentially high, involving data, metadata, and structures.

A multilayer view is fit for treating complex information entities by keeping contents properly organized within a unique framework. In fact, music information is made of heterogeneous facets, whose degree of abstraction may range from a purely logical definition in terms of symbols to the physical description of audio signals. Adopting the IEEE 1599 terminology, music information can be organized into the following layers: General, Logic, Structural, Notational, Performance, and Audio. Figure 2 shows the typical 6layer structure of an IEEE 1599 document, together with an example of contents. Please note that the multi-layer approach for music has been discussed in earlier works, such as [25], [26] and [27].

It is worth mentioning that not all layers are necessarily present for a given music piece. Of course, the higher is 
the number of available layers, the richer is the music description. For instance, many jazz pieces have no full score and a given performance could only be transcribed $a$ posteriori. Similarly, some folk songs or popular tunes do not present commonly-accepted metadata or instrumentation. Besides, no hierarchical relationship is implied among layers, since they show different facets of the same music piece.

The goal of layers is providing ad-hoc descriptions for the set of music events that constitute the score. Layers can contain 0,1 or $n$ descriptions of each music event. In order to support music coming from different traditions, cultures, geographical areas, historical periods it is necessary to adopt a format both powerful and flexible. In this sense, a key idea is defining the concept of music event in a loose way, as explained in the following.

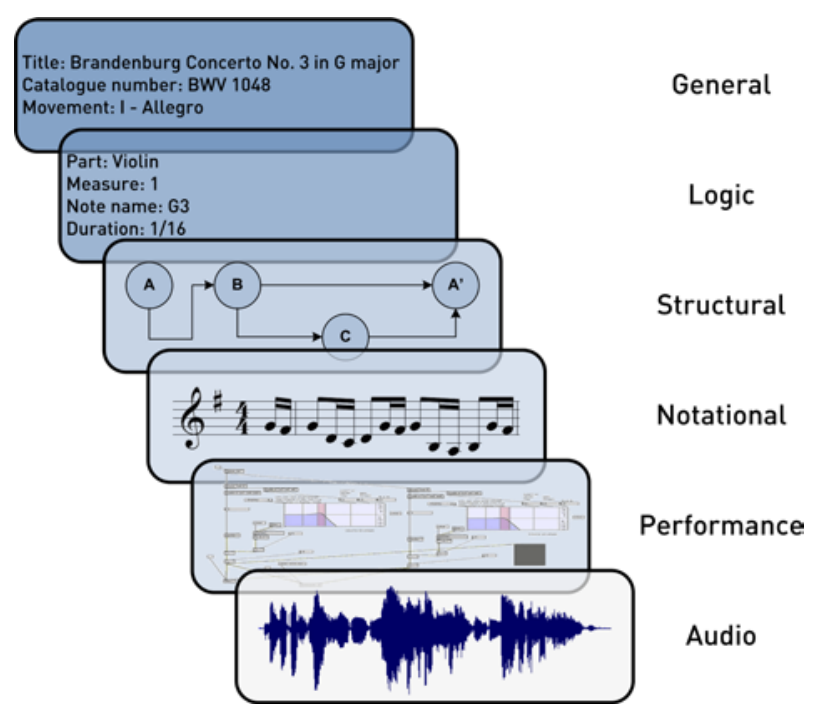

Figure 2. The IEEE 1599 6-layer structure

\subsection{The Spine}

The spine is the main data structure in an IEEE 1599 document, and its presence is mandatory for a file to be valid. Its purpose is listing music events and identifying them univocally by assigning unique labels.

In the official documentation, the concept of music event is left intentionally vague. In general terms, a music event can be defined as the occurrence of something that is considered important by the author of the encoding. For instance all the notes and rests of a score could be defined as music events: each symbol will be univocally identified and inserted into the spine. However, there are many other possibilities. For instance, in jazz music the only available form of score could be a harmonic grid, and music events could simply refer to the occurrence of chords. Similarly, in a musicological context, events of interest could be the occurrence of the fugue subject or the beginning of a dodecaphonic series. Please note that, thanks to the flexible definition of music event, neither traditional score notation nor a complete encoding of the piece are required to generate a valid IEEE 1599 document.

The spine is a sort of glue among IEEE 1599 layers, since the events listed and described inside different layers have to refer to the common event list provided by the spine itself. The name of this data structure recalls its function of "backbone" for the music work. This concept was first used in 1975 by D.A. Gomberg [28], who based a system for electronic music publishing on a similar structure, also called spine.

The spine simply lists events without defining them. For example, in a piece made of $n$ music events whatever they are - the spine would list $n$ entries without defining them from any point of view. The multiple and heterogeneous descriptions of events are demanded to the other layers.

\subsection{Synchronization among IEEE 1599 Layers}

If we consider the aforementioned 6-layer layout, the idea of heterogeneity seems to be merely related to the number of supported media types. But in addition each layer could contain many instances sharing the same kind of description or media type. For example, the Audio layer could contain different audio tracks, and the Structural layer could provide many different analyses for the same piece.

Each music event - univocally identified by the spine can be described in different layers (e.g. the graphical aspect of a note and its sound), as well as multiple times within a single layer (e.g. multiple music performances of the same event). Consequently, in the multilayer environment provided by IEEE 1599, one can recognize two synchronization modes:

- Inter-layer synchronization - It takes place among contents described in different layers, which have by definition heterogeneous data types. Applications involving multi-media and multi-modal fruition, such as score following, karaoke, didactic products, and multimedia presentations, can be realized thanks to this kind of synchronization;

- Intra-layer synchronization - It occurs among the contents of a single layer. Each layer contains - by definition - homogeneous information. Thanks to this feature, one can jump from an instance to another (e.g. from an audio performance to another) in real time, without losing synchronization.

By using the aforementioned kinds of synchronization, it is possible to design and implement advanced frameworks for music. In this context, the most relevant aspect is the possibility to integrate and synchronize heterogeneous kinds of description, including any form of meta-instrument notation. In other words, a particular music encoding - expressively conceived for a metainstrument - can interact with other kinds of notation and other media materials. The relevant consequences on music performance games and music education will become clear soon.

\section{The Proposed Educational Framework}

The goal of our research is fostering music education through the design and implementation of performance games based on the concept of meta-instrument and on the IEEE 1599 standard. On one hand, meta-instruments are currently the most expressive and challenging surrogates of real music instruments; on the other hand, the adoption of the IEEE 1599 standard allows countless didactic applications [29,30]. 
In our approach, two activities can be clearly distinguished: i) music encoding, and ii) music performance. Usually they are asynchronous, and the latter step follows in time the former one. For our goals music encoding implies the production of a "rich" IEEE 1599 document, namely a single XML file containing both the spine and the meta-instrument notation, mutually linked. Encoding music in a proper way during a live performance would be a hard task, even if theoretically feasible [31].

Needless to say, an IEEE 1599 document can contain much more, as explained in Section 3. For instance, the Audio layer could host a number of pre-recorded audio tracks referring to other performances of the piece, or conceived as a background for the current performance. Similarly, the Notational layer could host evocative graphics together with a traditional score in common Western notation.

With respect to other IEEE 1599 applications oriented to music education, here the novelty is the presence of meta-instrument notation. Usually meta-instruments require two kinds of input: performer's gesture and a custom notation to drive the system. Depending on the technical characteristics of the framework, notation may vary significantly. As an example, Figure 3 shows a Metapiano score excerpt containing basic symbolic information, i.e. notes, rests, and a few articulation signs.

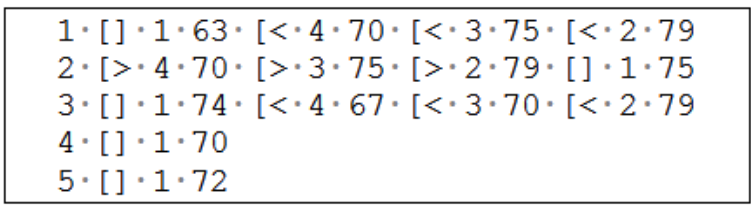

Figure 3. An example of plain-text notation for J. Haury's Metapiano. The score contains voice, pitch and velocity encoding, together with basic information on articulations

It is worth underlining that the information contained in a meta-instrument score is potentially redundant with the contents of the IEEE 1599 Logic layer, and actually the specifications of encoding rules make an automatic conversion among formats possible. Software tools and plug-ins have been developed to compile the Logic layer starting from commonly adopted formats (e.g. MusicXML and MIDI) as well as score editing software (e.g. MuseScore, MakeMusic Finale and Sibelius). Similarly, computer applications could be implemented to support custom meta-instrument scores, too.

Since IEEE 1599 supports any representation of score symbols, also new notation for music meta-instruments can be embedded and synchronized with all the other contents.

As regards the second phase - i.e. music performance before the design of this framework, two independent modules were available:

- An IEEE 1599 viewer, namely an environment oriented to a multi-layer and synchronized musical experience. This software is able to present simultaneously information contents from multiple layers, allowing the user to enjoy them together and to choose the material to bring to front. The user is active in the choice of current materials (scores, audio tracks, video clips, etc.), and he/she can use standard navigation controls (start, stop, pause, change current position); however, from the performance point of view, the user can only experience already prepared materials;

- A meta-instrument parser, where a symbolic score is loaded and the user can interact through the interface of the musical instrument. The parser is not standard, since it is customized for the specific metainstrument. Besides, it usually gets input only from the external controller and from a digital score representation. Consequently, other interactions with related materials are demanded to a posteriori processing of its output, which limits the expressive possibilities of the framework.

The idea is creating a unique framework where the two modules can be integrated, in order to create a performance game which takes advantage by both the approaches.

These two environments could be (and actually have been) implemented under different HW/SW architectures. Since IEEE 1599 is a standard, implementation details are not relevant for our proposal. For instance, IEEE 1599 players have been developed for multi-platform off-line fruition as well as embedded in Web portals. Similarly, there are some meta-instruments entirely implemented via software and others based on the communication between Arduino and Max/Msp environment. An example will be discussed in the final section of this paper as a case study. The music meta-instrument can be any hardware or software device capable of sending computer-interpretable messages: MIDI controllers, external peripherals such as computer keyboards, graphical interfaces, and so on.

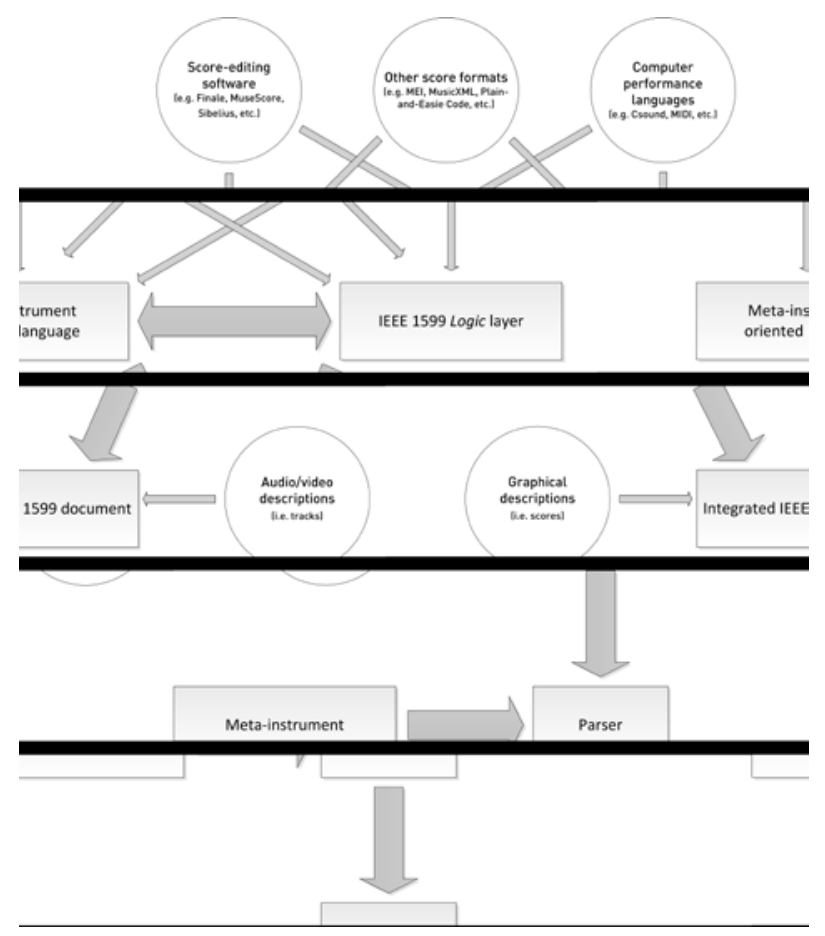

Figure 4. Flow chart of the proposed framework

In the proposed framework, the role of the parser is interpreting both the IEEE 1599 and the controller input, producing a sequence of commands to drive the player. One of the key roles is disambiguating synchronization. As mentioned before, most contents in an IEEE 1599 document have intrinsic timing information, such as all audio and video tracks. On the contrary, in this context metronome could be one of the parameters provided by the human player; in this case the parser has to match 
human gesture with meta-instrument notation, and other contents must be consequently timed.

Figure 4 illustrates the proposed framework. The upper half corresponds to music encoding, whereas the lower half represents music performance. The two approaches are matched by the parser, whose purpose is combining the information coming from the physical interface of the meta-instrument with the data contained in the IEEE 1599 document, now including also the meta-instrument score.

\section{Case Study: the Metapiano}

The Metapiano is a musical meta-instrument made of only nine piano keys that can be played with a few fingers, or even with only one finger [32]. This music metainstrument can produce music and sound by playing a limited number of keys. The musician can instantly interpret music with his/her own style by applying his/her rhythm, tempo, articulation, accent, dynamic and agogic phrasing. Figure shows the Metapiano's simplified interface.

The Metapiano notation stores only the notes to be performed by the player, encoding them according to the pianotechnie rules [33]. Thanks to the integration with IEEE 1599, now it is possible to provide a synchronized accompaniment too.

In this kind of meta-instrument pitch information is received from the score, and consequently reconstructed at parser level. The 9-key interface is provided only to allow more effective gestures. For instance, quick sequences of notes are easier to be obtained using many fingers, independently from the melodic contour. Similarly, a legato effect can be obtained only using at least two keys.

Experiments have shown that such an interface is extremely intuitive for complete beginners and inexpert players, who are not used to associate keys to sounds [34]. On the contrary, for skilled piano players this abstraction is harder to be managed. However, the latter category is not the typical recipient of a music performance game initiative.

In order to apply the IEEE 1599-based framework to the Metapiano case, a meta-instrument oriented language layer has been designed. This language is based on the syntactic and semantic encoding defined by Jean Haury. Starting from an XML encoding of the score, an integrated IEEE 1599 document is generated to feed the parser controlled by a musical meta-instrument interface. In this way, a 3-level hierarchy of music representation has been realized:

- A low-complexity encoding for complete beginners, namely people unable to read music scores and to play any music instrument;

- A medium-complexity encoding for learners, namely people who can read scores but with no instrumental skill;

- Finally, a high-level encoding for musicians, namely people interested in improving their music abilities and experiencing new kinds of music interfaces.

This approach can be easily ported to any other music meta-instrument, thanks to the extensibility of IEEE 1599 format. Currently, we are working on the software and hardware prototype of a meta-guitar, allowing the application of specific gestures and the control of typical guitar parameters. To this end, ad hoc custom notation is now under development.

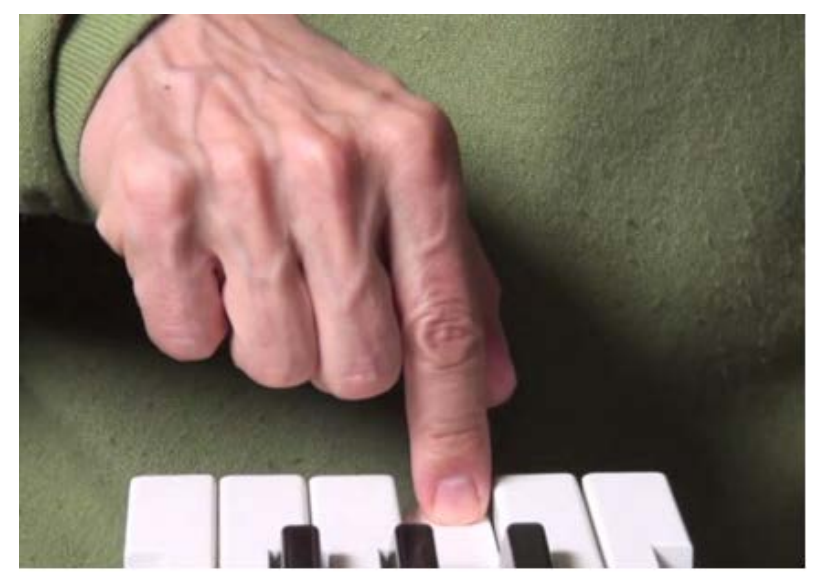

Figure 5. Jean Haury playing his 9-key Metapiano

\section{Conclusions and Future Work}

In this work we have critically analyzed the possibilities and the current limitations of performance games in music teaching. In our opinion, two coupled aspects can significantly enhance this didactic field: i) the adoption of meta-instruments, that are close to the expressiveness of real music instruments but simplify the approach of beginners or impaired people to instrumental practice, and ii) the employment of IEEE 1599 as a descriptive format for music, supporting multiple media encodings and synchronization. Even if commonly adopted for education purposes, this format was never used before for music performance games. The innovative idea is adopting a special controller acting as a meta-instrument to drive an IEEE 1599 parser/player.

Future work will concentrate on a re-engineered implementation of the framework, including the support for other meta-instruments. Besides, extensive tests will be conducted on young musicians and impaired children. As regards the test phase, an experimental project has been already conducted with visually impaired children in the context of the EMIPIU initiative. ${ }^{1}$ Two focus groups held in mixed classes demonstrated the validity of IEEE 1599-based applications in music education, also suggesting a number of improvements in the implementation. One of the consequences was the adoption of high-contrast color combinations in IEEE 1599 players, in order to improve score readability.

Next step will be stealth assessment in schools. Stealth assessment represents a quiet, yet powerful process by which learner performance data are continuously gathered during the course of playing/learning and inferences are made about the level of relevant competencies [35]. Stealth assessment is intended to support learning and maintain flow, defined as a state of optimal experience, where a person is so engaged in the activity at hand that self-consciousness disappears, sense of time is lost, and the person engages in complex, goal-directed activity not for external rewards, but simply for the exhilaration of doing [36]. Stealth assessment is also intended to remove (or seriously reduce) test anxiety, while not sacrificing

\footnotetext{
${ }^{1}$ Enhanced Music Interactive Platform for Internet User (EMIPIU), http://emipiu.di.unimi.it
} 
validity and reliability [37]. The goal is to eventually blur the distinction between assessment and learning [38]. This process - integrated in an educational context - allows the recognition by inference of specific abilities through performance games. The key assumptions of this approach are: i) learning by doing, typical of performance games, improves educational processes and outcomes; ii) different types of learning and learner attributes may be verified and measured during game play; iii) strengths and weaknesses of the learner may be analyzed to improve learning; and iv) feedback can be used to further support student learning and improve the technological framework.

Our validation strategy will be based on evidencecentered design [39] and experimentation in music institutions and schools. The IEEE 1599-based metainstrument framework will be constantly updated and improved in order to make it a more and more effective didactic tool.

\section{Acknowledgement}

The authors gratefully wish to acknowledge the researchers who contributed to this work. In particular, we want to thank for their support: Jean Haury, the inventor of the Metapiano, Pierre Castellotti, an expert in the field of meta-instruments and musician-to-machine interfaces, and the staff of Didael KTS S.r.l., who conducted early tests and organized focus groups.

\section{References}

[1] Finney, J., and Burnard, P., Music education with digital technology, Continuum, 2010.

[2] Mark, M., and Madura, P., Contemporary music education, Cengage Learning, 2013.

[3] Young, S., "Time-space structuring in spontaneous play on educational percussion instruments among three-and four-yearolds,” British Journal of Music Education, 20(1), 45-59, 2003.

[4] Barrett, A.M., "A Millennium Learning Goal for education post-2015: a question of outcomes or processes," Comparative Education, 47(1), 119-133, 2011.

[5] Jorgensen, E.R., "The aims of music education: A preliminary excursion,” Journal of Aesthetic Education, 31-49, 2002.

[6] Lillard, A.S., "Playful Learning and Montessori Education." American Journal of Play, 5(2), 2013.

[7] Munari, B., I laboratori tattili, Bologna: Zanichelli, 1985.

[8] Aiello, P., Di Tore, S., Di Tore, P.A., and Sibilio, M., "Didactics and Simplexity: Umwelt as a Perceptive Interface," Education Sciences \& Society, 4(1), 2013.

[9] Faiella F., and Mangione, G.R., E-learning. Le pratiche consolidate e i nuovi scenari di ricerca, Pensa Editore, 2012.

[10] Wechsler, D., "Intelligence defined and undefined: A relativistic appraisal," American Psychologist, 30(2), 135-139, Feb 1975.

[11] Denis, G., and Jouvelot, P., "Motivation-driven educational game design: applying best practices to music education," Proceedings of the 2005 ACM SIGCHI International Conference on Advances in computer entertainment technology, ACM, 462-465, 2005.

[12] Kim, Y.E., Schmidt, E.M., and Emelle, L., "MoodSwings: A Collaborative Game for Music Mood Label Collection,” ISMIR, 8, 231-236, 2008.

[13] Marczak, R., Robine, M., Desainte-Catherine, M., Allombert, A., Hanna, P., and Kurtag, G., "Enhancing expressive and technical performance in musical video games," Proceedings of the SMC, 2009.

[14] Miranda, E.R., and Wanderley, M.M., New digital musical instruments: control and interaction beyond the keyboard, AR Editions, Inc., 2006.

[15] Castellotti, P., and Malcangi, M., "Meta-instruments: the Musician-machine Interface and Gesture Sensing for Real-time
Algorithm Control,” DSP Application Day 2013, Maggioli Editore, 2013.

[16] Arfib, D., Couturier, J.M., and Kessous, L., "Expressiveness and digital musical instrument design," Journal of New Music Research, 34(1), 125-136, 2005.

[17] Baggi, D.L., and Haus, G., "IEEE 1599: Music Encoding and Interaction," IEEE Computer, 42(3), 84-87, 2009.

[18] Baggi, D.L., and Haus, G., Music Navigation with Symbols and Layers: Toward Content Browsing with IEEE 1599 XML Encoding, John Wiley \& Sons, 2013.

[19] Read, G., Music notation: A manual of modern practice, Taplinger Publishing Company, 1979.

[20] Parrish, C., The notation of medieval music, Pendragon Press, 1978.

[21] Kimiko, O., and Yoshihiko, T., "Ethnomusicology in Japan since 1970”, Yearbook for Traditional Music, 15, JSTOR, 155-162, 1983.

[22] Shirali, V., Sargam: an introduction to Indian music, Abhinav/Marg Publications, 1977.

[23] Rubsamen, W., "The earliest French lute tablature", Journal of the American Musicological Society, 21(3), 286-299, 1968.

[24] Serwadda, M., and Pantaleoni, H., "A possible notation for African dance drumming," African Music, 47-52, JSTOR, 1968.

[25] Lindsay, A., and Kriechbaum, W., "There's more than one way to hear it: multiple representations of music in MPEG-7," Journal of New Music Research, 28(4), 364-372, 1999.

[26] Steyn, J., "Framework for a music markup language," Proceedings of the First International IEEE Conference on Musical Application using XML (MAX2002), 22-29, IEEE, 2002.

[27] Haus, G., and Longari, M., "A multi-layered, time-based music description approach based on XML”, Computer Music Journal, 29(1), 70-85, 2005.

[28] Gomberg, D.A., "A computer-oriented system for music printing," Computers and the Humanities, 11(2), 63-80, 1977.

[29] Baratè, A., Haus, G., and Ludovico, L.A., "IEEE 1599: a New Standard for Music Education,” Proceedings of ELPUB 2009, 13th International Conference on Electronic Publishing: Rethinking Electronic Publishing, Innovation in Communication Paradigms and Technologies, 29-45, Edizioni Nuova Cultura, 2009.

[30] Baratè, A., and Ludovico, L.A., "New Frontiers in Music Education through the IEEE 1599 Standard," Proceedings of the 4th International Conference on Computer Supported Education (CSEDU 2012), 146-151, SciTePress - Science and Technology Publications, 2012.

[31] Baldan, S., Ludovico, L.A., and Mauro, D.A., "Algorithms for an Automatic Transcription of Live Music Performances into Symbolic Format," Auditory Display. Genesis of Meaning in Sound and Music. 6th International Symposium, CMMR/ICAD 2009, Copenhagen, Denmark, May 18-22, 2009: revised papers, LNCS, 5954, 422-437, Springer-Verlag, 2010.

[32] Haury, J., "The metapiano and the instant interpretation of musical scores,” DSP Application Day 2013, Maggioli Editore, 2013.

[33] Haury, J., "La grammaire de l'exécution musicale au clavier et le mouvement des touches," Analyse musicale: la musique et nous, 7, 10-26, 1987.

[34] Haury, J., and Schmutz, J., Développement d'un instrument d'interprétation de la musique pour personnes en situation de déficience motrice, Rapport APF, 2006.

[35] Shute, V.J., Ventura, M., Bauer, M., and Zapata-Rivera, D., "Melding the power of serious games and embedded assessment to monitor and foster learning," Serious games: Mechanisms and effects, 295-321, 2009.

[36] Csikszentmihalyi, M., Flow: The psychology of optical experience, New York: Harper Perrennial, 1990.

[37] Shute, V.J., Hansen, E.G., and Almond, R.G., "You Can't Fatten A Hog by Weighing It-Or Can You? Evaluating an Assessment for Learning System Called ACED," International Journal of Artificial Intelligence in Education, 18(4), 289-316, 2008.

[38] Shute, V.J., "Stealth assessment in computer-based games to support learning," Computer games and instruction, 55(2), 503524, 2011.

[39] Mislevy, R.J., Steinberg, L.S., and Almond, R.G., "On the structure of educational assessment," Measurement: Interdisciplinary Research and Perspective, 1(1), 3-62, 2003. 